\title{
Microstructural effect on the cavity expansion in a soil cylinder
}

\author{
J.D. Zhao, D.C. Sheng \& S.W. Sloan \\ Centre for Geotechnical and Materials Modelling, School of Engineering University of Newcastle, \\ Callaghan, NSW, Australia
}

\begin{abstract}
This paper presents an elastoplastic analysis for cavity expansion in a soil cylinder. Emphasis here is laid on the influence of microstructures in the soil on the macroscopic behaviour of the cavity expansion. In doing so, a strain gradient plasticity model is employed to model the soil behaviour in the cylinder. A numerical shooting method is developed to solve the resulting fourth-order ordinary differential equation with two-point boundary conditions for the gradient-dependent problem. Fully elastic-plastic solutions to the cavity expansion are obtained and they are compared with the corresponding conventional results. It is demonstrated that, in consideration of microstructural effects, the deformation and stress distributions in the cylinder are highly inhomogeneous during both the initial loading and the subsequent elastic and plastic expansion stages. The overall elastic-plastic behaviour of the gradient-dependent cylinder depends on the material parameters as well as the cylinder thickness. Interesting scale effects are observed and interpreted by the gradient-dependent model whereas this would be impossible by the corresponding conventional modeling. The results in this paper can be useful for benchmarking further numerical investigations of the cavity expansion problem.
\end{abstract}

\section{INTRODUCTION}

The expansion of spherical or cylindrical cavities in finite or infinite media has attracted much attention from both mechanics and material science communities. Owing to the simplicity of their geometry associated with cavity expansion problems, a number of closed-form solutions have been developed for various applications, such as metal indentation tests (Hill 1950), and practical geotechnical problems as pile installation, cone penetration tests (CPT) and pressuremeter tests (PMT) (e.g., Collins et al. 1992; Salgado et al. 1997, Yu 2000).

One important feature of geomaterials that has been largely neglected in previous cavity expansion studies is the effect of microstructure. Microstructures in soils and rocks in the form of fundamental material grains, microvoids, and microcracks influence the overall macroscopic behaviour of the material significantly during the deformation of the material body. The neglect of initial microstructure may, therefore, lead to remarkable discrepancies between observed material behaviour and theoretical predictions from constitutive models. Indeed, conventional continuum theory, on which most existing cavity expansion analyses have been based, does not attempt to address key geomaterial phenomena such as scale effects, strain localisation and catastrophic failures. To resolve these issues, it is now generally accepted that extra terms in the constitutive descriptions are needed to account for microstructural effects, via, for instance, various highorder constitutive relations (e.g., Cosserat \& Cosserat 1909; Toupin 1962; Mindlin 1964, 1965).

We hereby employ the Toupin-Mindlin strain gradient plasticity model to re-examine the cavity expansion problem in a soil cylinder, and to investigate the effect of microstructure on the deformation-stress and pressure-expansion relations. In consideration of extra gradient terms, the resultant differential governing equation becomes a fourth-order ordinary differential equation (ODE) with complex boundary conditions, which needs to be solved numerically as elegant analytical solutions are no longer obtainable. In this paper, we employ a numerical shooting approach, together with Broyden's iteration procedure, to solve this ODE for the elastic regime in the expanding cylinder. As for the gradient-dependent plastic expansion, the initial yielding position in the cylinder can not be known in advance. We first use the purely elastic case to find the point of initial yielding in the cylinder. Assumptions for the subsequent elastic-plastic cavity expansion behaviour are then made and examined a posterior using the numerical algorithms developed for the plastic solution. Extensive comparisons are made between the gradient-dependent results with those by the corresponding conventional theory. 


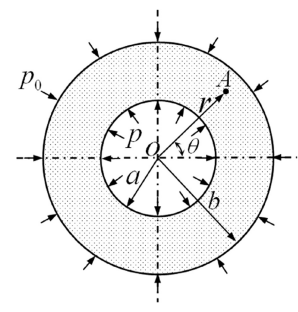

(a)

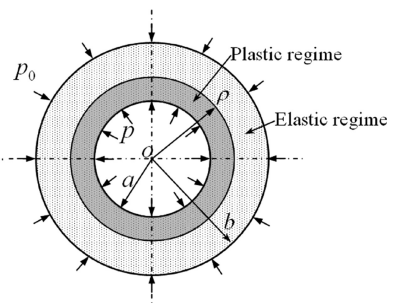

(b)
Figure 1. Schematic diagram of the cavity expansion in a gradient-dependent soil cylinder. (a) A purely elastic state; (b) A partly plastic state.

\section{CAVITY EXPANSION IN A GRADIENT- DEPENDENT SOIL CYLINDER}

\subsection{Problem description}

A cylindrical polar coordinate system is adopted, as shown in Fig.1. The soil cylinder is assumed to be initially free of stress. From this initial state, the hydrostatic pressure is slowly increased from zero to $p_{0}$ throughout the body, then the inner surface pressure is gradually increased from $p_{0}$ to $p$. This loading process is sufficiently slow so that it may be assumed to be quasi-static. Small strain deformation is assumed here for the sake of simplicity.

\subsection{Strain gradient plasticity model}

The soil is assumed to be homogeneous, isotropic and weightless, with its mechanical behaviour being characterised by a gradient-dependent elastic perfectlyplastic constitutive model as generalized from the linear elasticity strain gradient theory of Toupin (1962) and Mindlin (1965). In addition to the conventional Eulerian strains and Cauchy stresses, the strain gradients and their work-conjugate higher-order stresses are also assumed to be present in the material. The strains and strain gradients are respectively defined by:

$\varepsilon_{i j}=\frac{1}{2}\left(u_{i, j}+u_{j, i}\right), \quad \eta_{i j k}=\frac{1}{2}\left(u_{k, i j}+u_{k, j i}\right)$

Generalised plane strain conditions are assumed such that all the components of the strains and strain gradients associated with the out-of-plane coordinate (z) are zero. Due to the axi-symmetry of the problem, the displacement at a point $A$ of the cylinder can be reasonably assumed to be a function of $r$ only. Consequently, all the non-zero components of strains and strain gradients are related to $r$ by:

$\varepsilon_{r r}=u_{, r}, \varepsilon_{\theta \theta}=\frac{u}{r}, \eta_{r r r}=u_{, r r}, \eta_{\theta \theta r}=\frac{1}{r^{2}}\left(r u_{, r}-u\right)$,

$\eta_{r \theta \theta}=\eta_{\theta r \theta}=\frac{1}{r}\left(u_{, r}-\frac{u}{2 r}\right)$.
A generalised form of Hooke's law first proposed by Mindlin (1965) (see also, Zhao et al. 2007) is used to describe the isotropic linear elastic behavior of the gradient-dependent soil (the notation of tension positive is adopted):

$$
\begin{aligned}
& \left\{\begin{array}{l}
\sigma_{r r}=(\lambda+2 \mu) u_{, r}+\frac{\lambda}{r} u, \sigma_{\theta \theta}=\lambda u_{, r}+\frac{\lambda+2 \mu}{r} u, \\
\sigma_{z z}=\lambda\left(u_{, r}+\frac{u}{r}\right) . \\
\tau_{r r r}=c l^{2}\left(5 u_{, r r}+\frac{4}{r} u_{, r}-\frac{13}{4 r^{2}} u\right) \\
\tau_{r \theta \theta}=\tau_{\theta r \theta}=c l^{2}\left(\frac{3}{4} u_{, r r}+\frac{11}{4 r} u_{, r}-\frac{7}{4 r^{2}} u\right) \\
\tau_{r z z}=\tau_{z r z}=c l^{2}\left(\frac{3}{4} u_{, r r}+\frac{3}{4 r} u_{, r}-\frac{1}{2 r^{2}} u\right) \\
\tau_{\theta \theta r}=c l^{2}\left(\frac{3}{2} u_{, r r}+\frac{7}{2 r} u_{, r}-\frac{11}{4 r^{2}} u\right) \\
\tau_{z z r}=c l^{2}\left(\frac{3}{2} u_{, r r}+\frac{3}{2 r} u_{, r}-\frac{5}{4 r^{2}} u\right)
\end{array}\right.
\end{aligned}
$$

where $\lambda$ and $\mu$ are the conventional Lamé constants. In these equations, $l$ denotes an internal length scale resulting from the introduction of strain gradients and is related to the dimension of the microstructure in the material. The quantity $c$ denotes a single gradientdependent elastic parameter.

By neglecting body forces and considering the generalised plane strain condition, the following equilibrium equation for the radial direction of the cavity is found:

$$
\frac{\partial \sigma_{r r}^{*}}{\partial r}+\frac{1}{r}\left(\sigma_{r r}^{*}-\sigma_{\theta \theta}^{*}\right)=0
$$

where

$$
\left\{\begin{array}{l}
\sigma_{r r}^{*}=\sigma_{r r}-\left(\frac{\partial \tau_{r r r}}{\partial r}+\frac{1}{r}\left(\tau_{r r r}-\tau_{\theta \theta r}-\tau_{r \theta \theta}\right)\right) \\
\sigma_{\theta \theta}^{*}=\sigma_{\theta \theta}-\left(\frac{\partial \tau_{\theta r \theta}}{\partial r}+\frac{1}{r}\left(\tau_{\theta r \theta}+\tau_{r \theta \theta}+\tau_{\theta \theta r}\right)\right) \\
\sigma_{z z}^{*}=\sigma_{z z} .
\end{array}\right.
$$

If the three generalised stresses defined above satisfy the inequalities $\sigma_{\min }^{*} \leq \sigma_{\text {int }}^{*} \leq \sigma_{\max }^{*}$, the following generalised Tresca criterion is assumed to govern the yield behaviour of the material in the cylinder:

$f=\sigma_{\text {max }}^{*}-\sigma_{\text {min }}^{*}-\kappa=0$

where $\kappa$ denotes a cohesion parameter of the material. The relative magnitudes of $\sigma_{\theta \theta}^{*}, \sigma_{r r}^{*}$ and $\sigma_{z z}^{*}$ will be checked in the computations to determine which one 
corresponds to the maximum or minimum generalised principle stress.

\section{SOLUTION TO ELASTIC EXPANSION}

\subsection{Mathematic formulation and solution procedure}

Substitution of Eqs (3) and (4) into (6) and then into (5) results in the following fourth-order ODE for $u$ for the elastic expansion of the cavity:

$$
\begin{aligned}
& \dot{u}_{, r r r}-\frac{11}{5 r} \dot{u}_{, r r}-\left(\frac{61}{20 r^{2}}+\frac{(\lambda+2 \mu)}{5 c l^{2}}\right) \dot{u}_{, r r}+\left(\frac{51}{20 r^{3}}-\frac{(\lambda+2 \mu)}{5 c r l^{2}}\right) \dot{u}_{, r} \\
& -\left(\frac{51}{20 r^{4}}-\frac{(\lambda+2 \mu)}{5 c r^{2} l^{2}}\right) \dot{u}=0
\end{aligned}
$$

with the following boundary conditions:

$$
\left\{\begin{array}{l}
T_{r}(a)=\left.\left(-\sigma_{r r}^{*}+\frac{1}{r}\left(\tau_{r \theta \theta}-2 \tau_{r r r}\right)\right)\right|_{r=a}=p \\
R_{r}(a)=\left.\tau_{r r r}\right|_{r=a}=0 \\
T_{r}(b)=\left.\left(\sigma_{r r}^{*}+\frac{1}{r} \tau_{r \theta \theta}\right)\right|_{r=b}=-p_{0} \\
R_{r}(b)=\left.\tau_{r r r}\right|_{r=b}=0
\end{array}\right.
$$

In solving the problem constituted by (8) and (9), we employ a numerical shooting method together with Broyden's iteration procedure. A fourth-order explicit Runge-Kutta is used for solving the initial value problem for each iterating step (details may be referred to Zhao et al. 2006). The following parameters are used for the elastic analysis:

$a=1.0, \frac{b}{a}=2.0, \frac{\lambda}{\mu}=2.0, \frac{p_{0}}{\mu}=0.2, \frac{\Delta p_{s}}{p_{0}}=0.1$,

$\frac{c}{\mu}=1.0, \frac{l}{a}=0.1, \frac{\kappa}{p_{0}}=3.0$.

where $\Delta p_{s}$ denotes the increment size of pressure at the inner cavity surface. The proposed numerical procedure has been proved to be consistent, efficient and stable for the present problem.

\subsection{Numerical results}

Fig. 2 depicts the Cauchy stresses and generalised principal stresses in the cylinder for the gradientdependent case as compared to the conventional case. As is clearly shown, the conventional results for both $\sigma_{r r}$ and $\sigma_{\theta \theta}$ are homogeneous throughout the cylinder for this initial state, whereas the gradient theory values show a mildly inhomogeneous distribution.

It is interesting to investigate the sensitivity of the model response to some of the key parameters. We

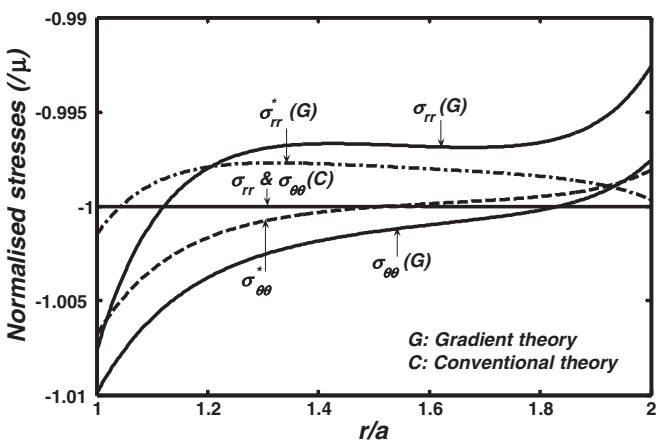

Figure 2. Distribution of normalized stresses in gradientdependent solid cylinder under a homogeneous hydrostatic pressure $p_{0}$.
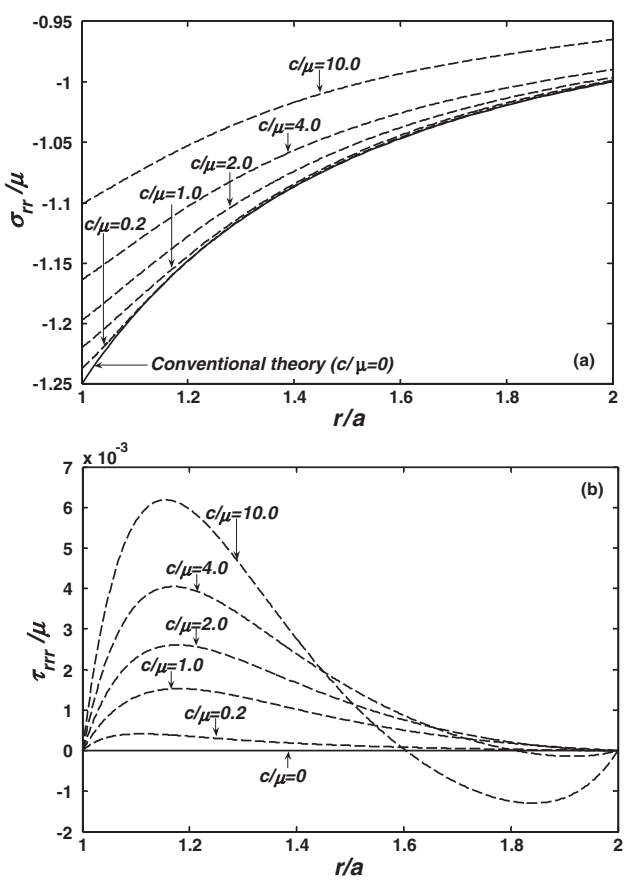

Figure 3. Sensitivity of normalized Cauchy stresses and higher stresses to $c / \mu$ at $p / p_{0}=1.25$.

choose an elastic expansion pressure of $p / p_{0}=1.25$. Fig. 3 presents the sensitivity of $\sigma_{r r}$ (Fig. 3a) and $\tau_{r r r}$ (Fig. 3b) to the variance of $c / \mu$. As can be seen, greater values of $c / \mu$ lead to the gradient-dependent stress distribution $\sigma_{r r}$ being above the conventional one. The influence of $c / \mu$ on the stresses $\tau_{r r r}$ is more pronounced. As expected, when $c / \mu=0$, the gradient effects vanish and the gradient-dependent curve for $\sigma_{r r}$ is identical to the conventional one, and $\tau_{r r r}$ tends to zero across the cylinder radius. Note that similar 

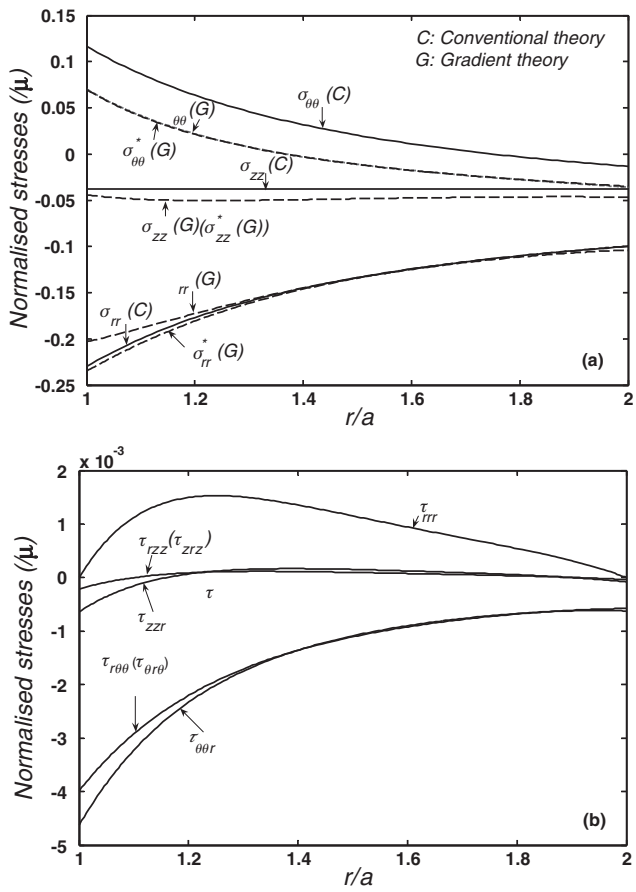

Figure 4. Variation of normalised Cauchy stresses, generalised stresses and high-order stresses across the cylinder for $p / p_{0}=2$.

observations for the influence of the internal length scale on the mechanical response can be found but omitted here for brief.

The distributions of normalised Cauchy stresses, generalised stresses and higher-order stresses at pressure level $p / p_{0}=2$ are presented in Fig. 4. As is shown in Fig. 4a, the magnitudes of the gradientdependent $\sigma_{z z}$ and $\sigma_{\theta \theta}$ are slightly smaller than those from conventional theory at this pressure. As for $\sigma_{r r}$, the gradient-dependent results are smaller than the corresponding conventional ones over the inner half of the radius, but become smaller in the outer half. This figure also shows the generalised stress $\sigma_{\theta \theta}^{*}$ is quite close to the gradient-dependent stress $\sigma_{\theta \theta}$, but smaller than the conventional one. Fig. $4 \mathrm{~b}$ shows the variation of highorder stresses in the cylinder. $\tau_{r r r}$ vanishes at both the inner and outer radii due to the high-order boundary conditions imposed by Eq. (9).

It is of particular interest to see from Fig. 4a that, under the current cavity pressure, $\sigma_{z z}^{*}$ is the intermediate generalised principal stress, with $\sigma_{\theta \theta}^{*}$ and $\sigma_{r r}^{*}$ being, respectively, the maximum and minimum ones. During the computations at other pressure levels in the elastic range, it was also found that the maximum value for $K=\left(\sigma_{\max }^{*}-\sigma_{\min }^{*}\right)$ always occur at the inner cavity wall. Therefore, the initial yielding will occur first at this surface. This finding will be used for the subsequent plastic expansion analysis.

\section{SOLUTION TO PLASTIC EXPANSION}

\subsection{Initial yielding}

Using the condition that initial yielding occurs at the inner cavity surface, we can accurately compute the initial yielding pressure $p_{c i}$. Upon initial yielding, the cylinder is still in an elastic state except at the inner cavity surface. Hence we have:

$\left\{\begin{array}{l}\left.\left(\sigma_{\theta \theta}^{*}-\sigma_{r r}^{*}\right)\right|_{r=a}=\kappa \\ \left.\tau_{r r r}\right|_{r=a}=0\end{array}\right.$

We then use these two new boundary conditions to replace the first two in Eq. (9), and carry out the numerical shooting method again to solve the displacement and stress field in the cylinder. On determining the stress field, the initial yielding pressure can also be obtained by the traction condition at $r=a$ :

$p_{c i}=T_{r}(a)=\left.\left[-\sigma_{r r}^{*}+\frac{1}{r} \tau_{r \theta \theta}\right]\right|_{r=a}$

In analogue to the conventional case it is assumed that, upon further loading, yielding will develop from the inner wall and spread outwards to the exterior wall in a progressive manner. At any intermediate state of this process, the solid cylinder is said to be partly plastic. Fig. $1 \mathrm{~b}$ depicts a cylinder experiencing partly plastic deformation.

\subsection{Partly plastic state}

In conventional mechanics, the assumptions of small strain and a Tresca yield criterion render the stress field in the cylinder statically determinate, and it is possible to derive the Cauchy stresses merely by knowing $\rho$ and the cavity pressure $p$ without needing to calculate the deformation in the plastic region (e.g., Hill 1950). For the gradient-dependent model treated here, the problem is too complicated to be analytically solvable. The key difficulty is that the stress field in the plastic region is not statically determinate because of the number of unknown state variables and the nature of the boundary conditions. In dealing with these problems, we fully employ the aforementioned elastic-plastic boundary assumption for the partly plastic case, and develop a systematic numerical procedure for solving the stress distribution and cavity pressure-outer wall displacement curve (see Zhao et al. 2006 for details). However, we do not intend to determine the displacements in the plastic regimes as additional complex assumptions regarding the plastic flow rule are required which will make the problem too complicated to solve. 

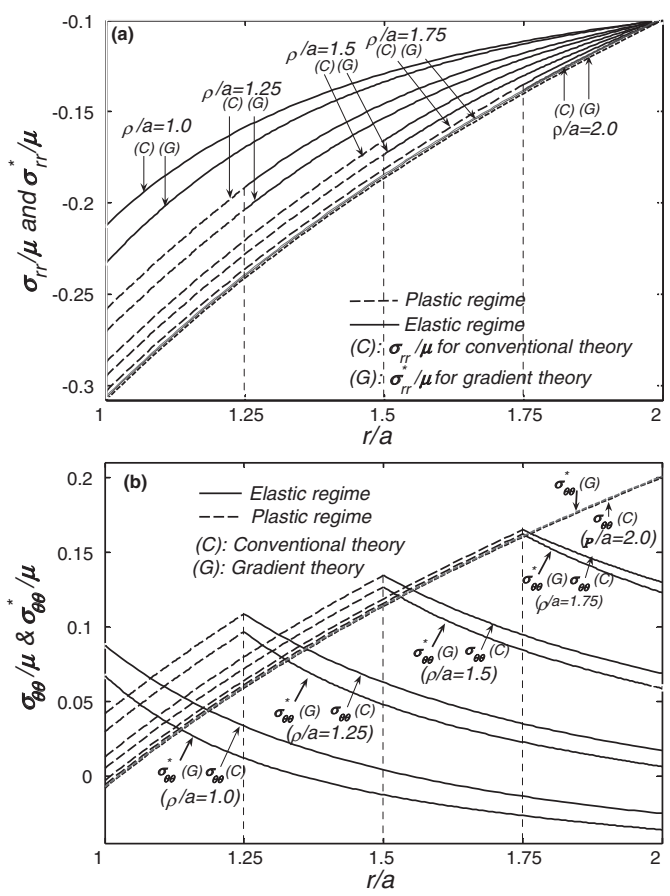

Figure 5. Variations of the normalized stresses $\sigma_{r r}^{*}$ and $\sigma_{\theta \theta}^{*}$ as a function of the plastic radius. (' $\mathrm{G}$ ' denotes gradient theory; ' $\mathrm{C}$ ' denotes conventional theory).

\subsection{Fully plastic state}

When the plastic zone reaches the outer boundary and the cylinder is in a fully plastic state, and the corresponding pressure is assumed to be $p_{c r}$. At this critical state, we assume that, for any point on the outer surface of the cylinder, the elastic relations still apply and the stresses must satisfy the yield condition. This fully plastic problem can then be treated as a special case of the partly plastic expansion, using the adapted numerical shooting procedure proposed in Section 4.2.

\section{$4.4 \quad$ Numerical results}

We use the same parameters as presented in Eq. (10) for the plastic expansion analysis. Semi-analytical solution to the plastic expansion is obtained. Fig. 5 illustrates the variation in the normalised stresses $\sigma_{r r}^{*}$ and $\sigma_{\theta \theta}^{*}$ across the cylinder wall as a function of the plastic radius $\rho$. It is shown shows in Fig. 5a that the elastic and plastic curves for $\sigma_{r r}^{*}$ are smoothly continuous at the plastic regime boundary $\rho$ and, before the fully plastic stage is reached, are smaller than the corresponding conventional stress $\sigma_{r r}$ at any point of the cylinder. At the onset of fully plastic
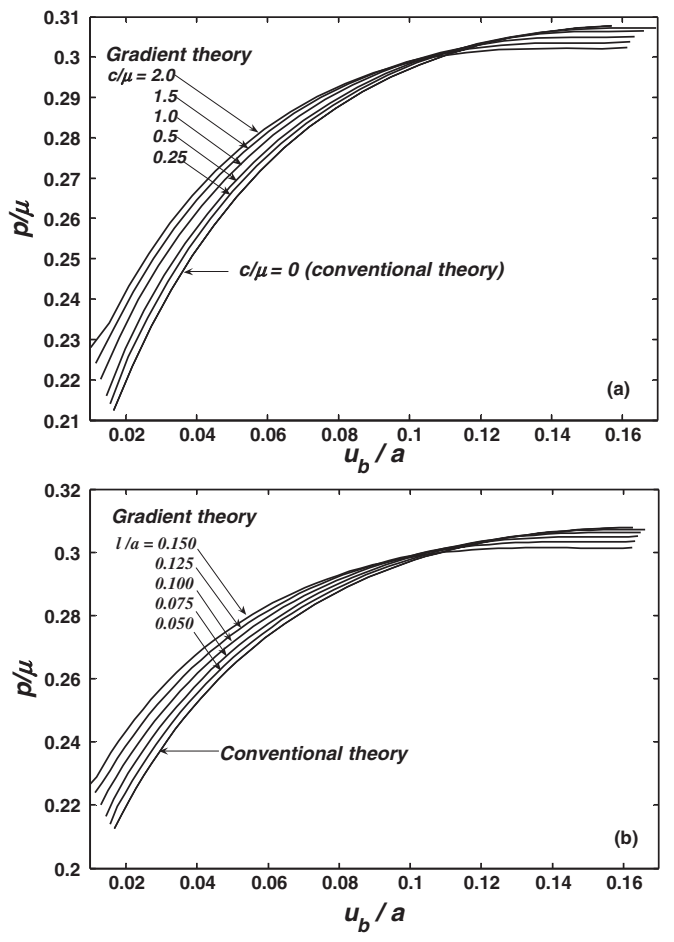

Figure 6. Sensitivity studies for the pressure-expansion relation for plastic loading of a gradient-dependent solid, (a) to $c / \mu(l / a=0.1)$; (b) to $l / a(c / \mu=1.0)$.

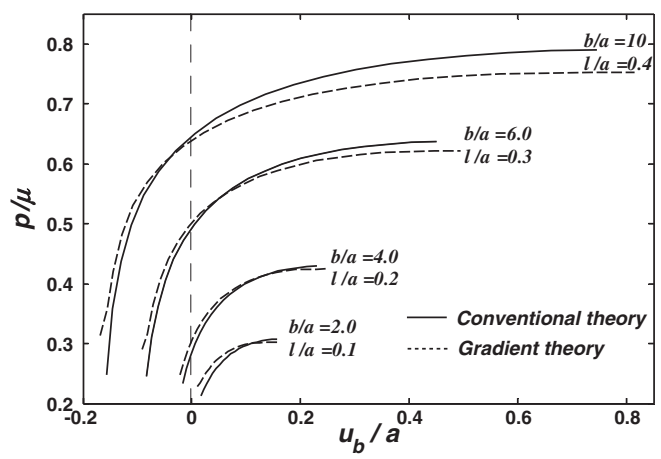

Figure 7. Pressure-expansion relations for gradientdependent solid cylinders of varying thickness.

deformation, however, $\sigma_{r r}^{*}$ is slightly larger than $\sigma_{r r}$. Fig. $5 \mathrm{~b}$ shows that the stress $\sigma_{\theta \theta}^{*}$ in the elastic and plastic regimes is still continuous at the plastic radius $\rho$ but non-smooth. This mirrors the behaviour from conventional theory for $\sigma_{\theta \theta}$. It is also evident that, prior to the onset of any plastic deformation, the generalised stress $\sigma_{\theta \theta}^{*}$ is always smaller than its conventional counterpart $\sigma_{\theta \theta}$. At the onset of fully plastic deformation, however, 
$\sigma_{\theta \theta}^{*}$ and the conventional stress $\sigma_{\theta \theta}$ are quite similar, with $\sigma_{\theta \theta}^{*}$ being slightly greater than $\sigma_{\theta \theta}$.

It is confirmed that during all stages of plastic expansion $\sigma_{z z}^{*}$ remains the intermediate stress of the three, while $\sigma_{\theta \theta}^{*}$ is the maximum. It is also found that the difference $\sigma_{\theta \theta}^{*}-\sigma_{r r}^{*}$, for the elastic regime in the cylinder, is always a maximum value at the elastic-plastic interface, which implies that, whenever further plastic yielding occurs, it always happens at this interface so that the plastic regime will develop in a progressive manner from the inner cavity radius to the outer cavity radius. This matches the assumptions made $a$ priori in the analysis.

Fig. 6 shows the influence of $c / \mu$ and $l / a$ on the plastic pressure-displacement relation in terms of the cavity pressure and the displacement at the outer surface several. Compared with the conventional curve, the gradient-dependent $p-u$ responses are generally stiffer but have a slightly lower ultimate pressure at the fully plastic state for both cases. Since smaller value of $c / \mu$ imply that the effects of the gradient terms are less significant and small value of $l / a$ implies the microstructures are negligible, these results are as expected.

We further present in Fig. 7 the pressure-expansion responses for cylinders with various thicknesses but with similar shape. As expected, the thicker cylinders have the highest ultimate cavity pressures. As the cylinder thickness increases, the elastic stiffening effect caused by the gradient-dependent terms becomes less pronounced. This is to say, in a thick cylinder, the stiffening effects from the elastic region decay more quickly than in a thin cylinder. For example, for the case of $b / a=10$, the weakening effects in the plastic region outweigh the stiffening effects from the elastic region when $1 / 7$ of the cylinder is still elastic. In contrast, for the case of $b / a=2$, this transition occurs when only a small elastic area is left in the cylinder $(\rho \approx b / 30)$. This phenomenon can be interpreted as a size effect for the cylindrical expansion problem, and is only discernable by the use of gradient theory.

\section{CONCLUSIONS AND DISCUSSIONS}

This paper has investigated cylindrical cavity expansion in an elastoplastic gradient-dependent media. The strain gradient plasticity model assumes small strains and is used in combination with a generalised form of Tresca's yield criterion. A numerical shooting method and Broyden's iteration procedure are used to solve the governing fourth-order ODE equation system with two-point boundary conditions. The elastic and plastic solutions obtained have been compared extensively against those derived from conventional analysis. It is shown that the stress distribution in the cylinder for the gradient-dependent case is highly inhomogeneous, even under a uniform hydrostatic pressure at the initial state. The influence of microstructure on the stress and deformation during the cavity expansion process can be modelled by adjusting the gradient-dependent elastic modulus and the internal length scale. When these quantities are large, the cavity expansion solutions exhibit pronounced differences from those obtained by conventional theory. The effect of the cylinder thickness on the obtained the pressure-expansion relations is also clearly depicted by this analysis.

It is worth noting that the proposed numerical procedure for solving the gradient-dependent cavity expansion problem cannot be used to simulate the case of cavity expanding from a zero radius via a similarity solution. This is because all the length variables in the present analysis have been normalised with respect to the inner cavity radius. In this case, the cylinder thickness could be used as a reference for the normalisation. Moreover, the current solution to the plastic expansion case is not a closed form one, as the deformation in the plastic regime has not been obtained. Where possible, it is preferable for theoretical studies to be benchmarked against experimental data. This is not done in the present work because, in contrast to various conventional theories, gradient-enhanced theories have so far received relatively little calibration against real data due to their recent development. Indeed, many of their parameters are still an open question. Nevertheless, we suggest that the semi-analytical solutions for cavity expansion in a higher order continuum presented here can be used for benchmarking of any similar studies in the future.

\section{REFERENCES}

Collins, I.F., Pender, M., Wang, Y., 1992. Cavity expansion in sands under drained loading conditions. Int. J. Numer. Analy. Meth. Geomech., 16(1), 3-23.

Cosserat, E., Cosserat, F., 1909. Théorie des Corps Déformables. Hermann A. et Files, Paris.

Hill, R., 1950. The Mathematical Theory of Plasticity. Oxford University Press, London.

Mindlin, R.D., 1964. Micro-structure in linear elasticity. Arch. Ration. Mech. Anal. 16, 51-78.

Mindlin, R.D., 1965. Second gradient of strain and surface tension in linear elasticity. Int. J. Solids Structures 28, $845-857$.

Salgado, R., Mitchell, J.K., Jamiolkowski, M., 1997. Cavity expansion and penetration resistance in sand. J. Geotech. Geoenviron. Eng., ASCE, 123(4), 344-354.

Toupin, R.A., 1962. Elastic materials with couple stresses. Arch. Ration. Mech. Anal. 11, 385-414.

Yu, H.S., 2000. Cavity Expansion Methods in Geomechanics. Kluwer Academic Publisher.

Zhao, J.D., Sheng, D.C., Sloan, S.W. 2006. Cavity expansion of a gradient solid cylinder. Int. J. Solids Structures (available online: doi:10.1016/j.ijsolstr.2006.11.023)

Zhao, J.D., Sheng, D.C., Sloan, S.W., Krabbenhoft, K., 2007. Limit theorems for gradient-dependent elastoplastic geomaterials. Int. J. Solids Structures 44, 480-506. 